\title{
Bcl-2 family: Novel insight into individualized therapy for ovarian cancer (Review)
}

\author{
JING YUAN, HUA LAN, XIAOYAN JIANG, DA ZENG and SONGSHU XIAO \\ Department of Gynecology and Obstetrics, Third Xiangya Hospital, \\ Central South University, Changsha, Hunan 410013, P.R. China
}

Received March 10, 2020; Accepted June 25, 2020

DOI: $10.3892 /$ ijmm.2020.4689

\begin{abstract}
Chemoresistance to platinum-based chemotherapy for ovarian cancer in the advanced stage remains a formidable concern clinically. Increasing evidence has revealed that apoptosis represents the terminal events of the anti-tumor mechanisms of a number of chemical drugs and has a close association with chemoresistance in ovarian cancer. The B-cell lymphoma-2 (Bcl-2) family plays a crucial role in apoptosis and has a close association with chemoresistance in ovarian cancer. Some drugs that target Bcl-2 family members have shown efficacy in overcoming the chemoresistance of ovarian cancer. A BH3 profiling assay was found to be able to predict how primed a cell is when treated with antitumor drugs. The present review summarizes the role of the Bcl-2 family in mediating cell death in response to antitumor drugs and novel drugs that target Bcl-2 family members. The application of the new functional assay, BH3 profiling, is also discussed herein. Furthermore, the present review presents the hypothesis that targeting Bcl-2 family members may prove to be helpful for the individualized therapy of ovarian cancer in clinical practice and in laboratory research.
\end{abstract}

\section{Contents}

1. Introduction

2. Central role of the Bcl-2 family in the apoptotic pathway

3. Concise mechanisms of platinum-based chemotherapy

4. Bcl-2 family members in ovarian cancer

5. Bcl-protein inhibitors and ovarian cancer

6. BH3 profiling and ovarian cancer

7. Conclusion

Correspondence to: Dr Songshu Xiao, Department of Gynecology and Obstetrics, Third Xiangya Hospital, Central South University, 138 Tongzipo Road, Yuelu, Changsha, Hunan 410013, P.R. China E-mail: xiaosongshu@csu.edu.cn

Key words: Bcl-2 family, apoptosis, BH3 profiling, ovarian cancer, individualized therapy

\section{Introduction}

Ovarian cancer ranks as the third leading type of cancer among all the gynecological cancers, with a very high mortality rate according to the latest report by the International Agency for Research on Cancer (IARC) $(1,2)$. The majority of patients are diagnosed at an advanced stage and there is no curative therapy (3). The most common therapy for advanced stage disease includes maximal cytoreductive surgery and platinum/taxane-based chemotherapy, which always attains an initial response $(4,5)$. Over the past 20 years, this combination has become the standard of care for patients with ovarian cancer (6). Although $70 \%$ of ovarian cancer patients exhibit an effective response to the first application of platinum chemotherapy (7), the majority (80\%) will relapse within 6 months, and this has been classified as 'platinum resistance' $(8,9)$, and is one of the major reasons for the high clinical mortality rate of patients with ovarian cancer (10). Therefore, chemoresistance has become an urgent issue that needs to be resolved in clinical practice, encouraging the identification of more effective drugs. Poly ADP ribose polymer (PARP) inhibitors play an antitumor role by inhibiting DNA repair and promoting tumor cell apoptosis (11). At present, some clinical trials have found that PARP combined with other anticancer agents, such as carboplatin and paclitaxel, can improve the prognosis of patients with recurrent drug-resistant ovarian cancer (12).

Apoptosis is critical for regulating cellular homeostasis. The dysfunction of apoptosis is one of the characteristics of cancer and other diseases (13). Therefore, most conventional chemotherapeutic agents, such as cisplatin, confer an antitumor effects by mainly relying on the activation of the apoptosis of cancer cells (14). As significant regulators of apoptosis, the B-cell lymphoma-2 ( $\mathrm{Bcl}-2)$ family is closely related to the chemoresistance of ovarian cancer. Additionally, cancer cells can evade apoptosis in multiple pathways to achieve immortalization and the evasion of apoptosis is a significant mechanism of the chemoresistance of cancer cells $(15,16)$. BH3 profiling is a functional assay that can measure how primed a cell is to execute apoptosis by detecting the release of pro-apoptotic factors. This assay was proposed to solve the clinical issue of neoplastic hematological disorders due to the high heterogeneity and chemoresistance rate of the cells (17) and may be of assistance in overcoming chemoresistance in ovarian cancer. 
In the following chapters, the central role of Bcl-2 family members in the apoptotic pathway and the mechanisms through which they regulate apoptosis in ovarian cancer in response to antitumor agents, such as platinum, will be discussed. The present review also discusses the clinical function of a novel assay, $\mathrm{BH} 3$ profiling.

\section{Central role of the Bcl-2 family in the apoptotic pathway}

Apoptosis, also known as programmed cell death, is a programmed biological activity with distinct genetic and epigenetic pathways that involves a form or cellular suicide without triggering inflammatory responses, which is the main characteristic differentiating the process from necrosis $(18,19)$. From a molecular mechanistic aspect, apoptosis can be activated by 3 main signaling pathways: The extrinsic pathway, intrinsic apoptosis and endoplasmic reticulum (ER) stress-induced apoptosis (20). Additionally, some other apoptotic pathways have been found, such as the granzyme B pathway (21). It seems that these pathways are irrelevant; however, they can actually be linked through Bcl-2 family proteins. Research on the mechanisms of apoptosis in cells has shed light on the fact that the Bcl-2 family plays a central role in the apoptotic pathway (22) (Fig. 1). The threshold of cell fate is mediated by the balance between pro-survival and pro-apoptotic Bcl-2 family members (23). This basic interaction is conserved from sponges to humans $(24,25)$. The specific regulatory mechanisms of these apoptotic signaling pathways differ from body to body. However, all of these mechanisms eventually return to the loss of pro-survival activity and the gain of pro-apoptotic activity, which is why the interactions of this family are explored and their regulatory function network is clarified (26).

To date, at least 16 members of this family that contain Bcl-2 homology $(\mathrm{BH})$ domains have been identified; these proteins can be further sorted into 3 major groups (Table I). The first group is the proteins that contain only one $\mathrm{BH}$ domain, termed BH3-only proteins, such as BID and BIM, which function as apoptotic promoters. BH3-only proteins are activated earlier than any of other proteins involved in this pathway; thus, they act as molecular sentinels. The existence of the BH3 motif is a key feature of pro-apoptotic proteins, and it is necessary for pro-apoptotic activity $(27,28)$. The second group is composed of the pro-apoptotic proteins that contain $3 \mathrm{BH}$ domains (BH1, BH2 and $\mathrm{BH} 3)$, such as BAX and BAK. The third group includes anti-apoptotic proteins, such as Bcl-2, Bcl-xL and Mcl-1, which contains 4 $\mathrm{BH}$ domains (BH1, BH2, BH3 and $\mathrm{BH} 4)$. They can protect cells from apoptosis by sequestering their pro-apoptotic counterparts (19,29-32).

After a variety of stimuli reach cells to induce apoptosis, the Bcl-2 family is eventually activated as multiple proteins and pathways converge upon the Bcl-2 family (29). It has been widely postulated that the majority of Bcl-2 family proteins are located in the nuclear envelope, the ER and the outer mitochondrial membrane (OMM). These characteristic locations are consistent with the function of Bcl-2 family proteins. However, the role of the Bcl-2 family in OMM is the core mechanism of intrinsic apoptosis (26). Exactly as is described, mutant Bcl-2 family proteins that are disabled to anchor the membrane have been found to be less effective at preventing apoptosis in some systems (31).

As molecular sentinels, BH3-only proteins, such as BID and $\mathrm{BIM}$ are the first members to be activated. There is evidence to suggest that $\mathrm{BH} 3$-only proteins can directly interact with and activate BAX/BAK $(33,34)$. There is an activation priority existing between these proteins: BID first activates BAK, while BIM first activates BAX (35). Activated pro-apoptotic proteins translocate to the mitochondrion and disrupt the integrity of the OMM in a process termed mitochondrial outer membrane permeabilization (MOMP). The exact mechanism of this process remains unclear (36). Certain studies have suggested an interesting hypothesis in which activated BAX undergoes oligomerization at the mitochondrial membrane, forming rings, lines and incomplete rings or arc structures of different shapes and sizes, resulting in the mitochondrial inner membrane remodeling to facilitate the release of cytochrome $c(37,38)$, and subsequently activating the caspase cascade (26). Another possible mechanism is that Bcl-2 family pro-apoptotic proteins can break the stable state of the $\mathrm{Ca}^{2+}$ level within the ER and then increase the $\mathrm{Ca}^{2+}$ transfer to the mitochondrion, which leads to mitochondrial swelling and then to the perturbation or rupture of the OMM, resulting in mitochondrial dysfunction and the release of pro-apoptotic molecules into the cytosol (39).

A crucial component that cannot be ignored is the mitochondrion intermembrane space (IMS), where the majority of pro-apoptotic factors are located (32). Once the OMM is disrupted, pro-apoptotic proteins release from the IMS into the cytoplasm in response to apoptotic signals, such as DNA damage, oxidative stress, ER stress and other events (31). As soon as these pro-apoptotic factors are released into the cytosol, they can initiate different cascades, leading to apoptosis and eventually causing cell death. All of the functional factors, such as cytochrome $c$, apoptosis protease activating factor 1 (Apaf-1), dATP and procaspase-9, assemble together and interact with each other, forming the apoptosome. This structure favors procaspase- 9 rapidly converting into caspase-9, thereby eventually killing the cell (31).

\section{Concise mechanisms of platinum-based chemotherapy}

Platinum has been an indispensable antitumor agent for almost 40 years, since it was first approved in the USA by the Food and Drug Administration (FDA) in 1978 for the treatment of testicular, bladder and ovarian cancers (40). As platinum plays an increasing role in the treatment of cancers, such as ovarian cancer, the elucidation of the mechanisms through which platinum and its analogues function in normal and cancer cells has become imperative. However, the exact functional mechanisms of chemotherapeutic drugs have not been fully elucidated.

Briefly, there are 4 steps that platinum undergoes to function as an antitumor agent. First, drugs need to enter to cells through passive diffusion or active transport. In this step, some transporters involved in the cellular uptake of platinum and its derivatives have been identified, such as copper transporter 1 , which is considered as the major influx transporter for platinum drugs (41). Pan et al found that theaflavin-3,3'-digallate (TF3), a black tea polyphenol, 


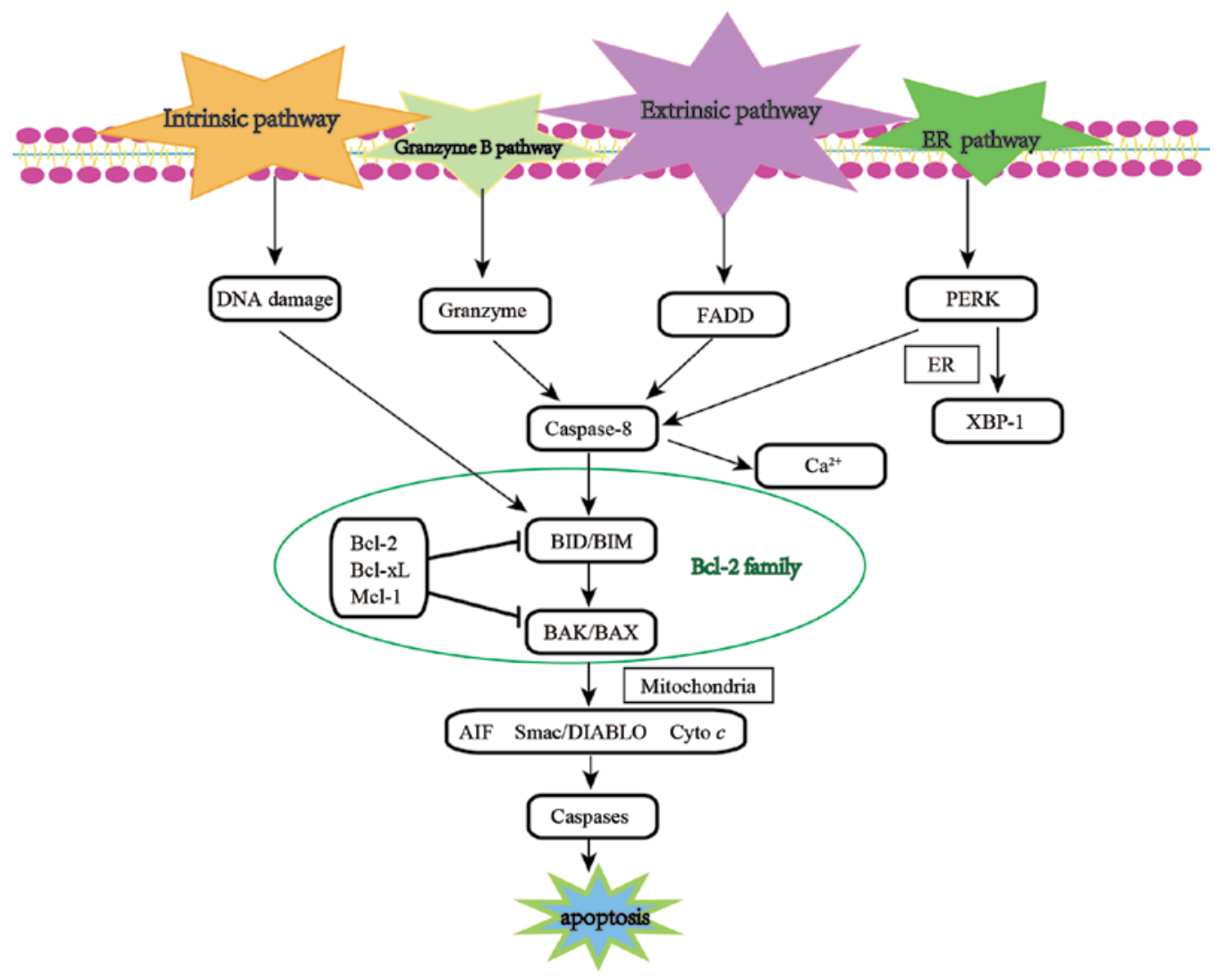

Figure 1. Overview of the signaling pathway of apoptosis. The diagram illustrates the main proteins and 4 signaling pathways mediating cell apoptosis. These are: i) The intrinsic pathway; ii) the granzyme B pathway; iii) the extrinsic pathway; and iv) the endoplasmic reticulum (ER) stress-induced apoptotic pathway. The 4 pathways converge on the mitochondrion, and Bcl-2 family members play a central in regulating apoptosis among all the four pathways. ER, endoplasmic reticulum; FADD, fas-associating protein with a novel death domain; PERK, protein kinase R-like ER kinase; XBP-1, X-box binding protein 1; AIF, apoptosis-inducing factor; Smac/DIABLO, Second mitochondrial activator of caspases/direct IAP-binding protein with low PI; Cyto $c$, cytochrome $c$; BID, BH3-interacting domain; BIM, Bcl-2-interacting protein; BAK, Bcl-2 antagonist killer 1; BAX, Bcl-2 associated X Protein; Bcl-2, B cell lymphoma protein 2; Bcl-xL, Bcl-2-related protein long form of Bcl-x; Mcl-1, myeloid cell leukaemia-1.

upregulated the expression of copper transporter 1 to enhance the cytotoxicity of platinum in ovarian cancer (42). Once entering the cell, platinum will undergo a substitution reaction in which a water molecule replaces the chloride as a ligand, which is conducive to platinum binding to DNA and causing DNA damage by forming stable Pt-DNA crosslinks, which will destabilize the structure and function of the DNA. Due to the DNA repair system, however, cells can survive from agent attack. Alternatively, cells that cannot repair the DNA lesion will activate several signaling pathways, including the p53-mediated signaling pathway $(43,44)$, the Myc gene-mediated signaling pathway $(45,46)$ and the PI3K/Akt/mTOR signaling pathway $(47,48)$. The activation of these signaling pathways will affect the levels of Bcl-2 family proteins and ultimately lead to apoptosis, which functions as the terminal event in chemotherapy (Fig. 2). Chemoresistance serves as a crucial reason for a poor prognosis and a high mortality of ovarian cancer. However, these factors mentioned above do not completely describe the full biological repertoire of the resistance mechanism to platinum in ovarian cancer.

\section{Bcl-2 family members in ovarian cancer}

As the induction of cell apoptosis is the terminal event in platinum-based chemotherapy, resistance to apoptosis is one of the crucial labels of resistance to chemotherapy (49). There are a number of cancer cells that evade apoptosis. Preventing cancer cells from evading apoptosis is a promising strategy with which to reverse resistance to chemotherapy. The Bcl-2 family, as a central part of the apoptotic signaling network, plays a vital role in the resistance of ovarian cancer to chemotherapy. The Bcl-2 family members were initially discovered to be hallmarks of follicular lymphomas (50). There is a sophisticated interaction network between Bcl-2 family members that has yet to be completely known (51). However, in cancer cells, the interaction among Bcl-2 family members may not be the same as in normal cells and there is ample evidence to indicate that a number of cancer cell lines present a disrupted Bcl-2 protein expression that is associated with cancer survival and chemoresistance.

Bcl-2 pro-survival proteins. Ovarian cancer, similar to other tumors, has been shown to overexpress Bcl-2 and its family members (52-55). Of note, although Bcl-2 pro-survival proteins can prolong the survival period of tumor cells in hematological tumors, playing an important role in tumorigenesis (54), the truth seems to be opposite in ovarian cancer cell lines. Although the expression of Bcl-2 family proteins in ovarian cancer cells and tissues has not yet been fully elucidated, studies have confirmed that Bcl-2 family proteins 
Table I. Bcl-2 family members.

\begin{tabular}{|c|c|c|c|}
\hline Name & BH domain & Members & Function \\
\hline BH3-only proteins & $\mathrm{BH} 3$ & $\begin{array}{l}\text { BID, BIM, NOXA, } \\
\text { PUMA, BIK, BAD, BF, HRK }\end{array}$ & $\begin{array}{l}\text { Initiators of apoptosis: Proteins that is first } \\
\text { activated during the initiation of apoptosis. }\end{array}$ \\
\hline Pro-apoptotic proteins & BH1, BH2, BH3 & $\mathrm{BAX}, \mathrm{BAK}, \mathrm{BOK}$ & $\begin{array}{l}\text { Executors of apoptosis: Proteins that can } \\
\text { change the permeability of mitochondrial } \\
\text { membrane by aggregating into the outer } \\
\text { membrane of mitochondria to form oligomers. }\end{array}$ \\
\hline Anti-apoptotic proteins & $\begin{array}{l}\mathrm{BH} 1, \mathrm{BH} 2, \mathrm{BH} 3, \\
\mathrm{BH} 4\end{array}$ & $\begin{array}{l}\text { Bcl-2, Bcl-xL, Mcl-1, Bcl-w, } \\
\text { A1/BFL-1 }\end{array}$ & $\begin{array}{l}\text { Antagonists of apoptosis: Proteins that } \\
\text { inhibit the aggregation of proapoptotic } \\
\text { proteins to prevent the initiation of apoptosis. }\end{array}$ \\
\hline
\end{tabular}

BH, Bcl-2 homology.

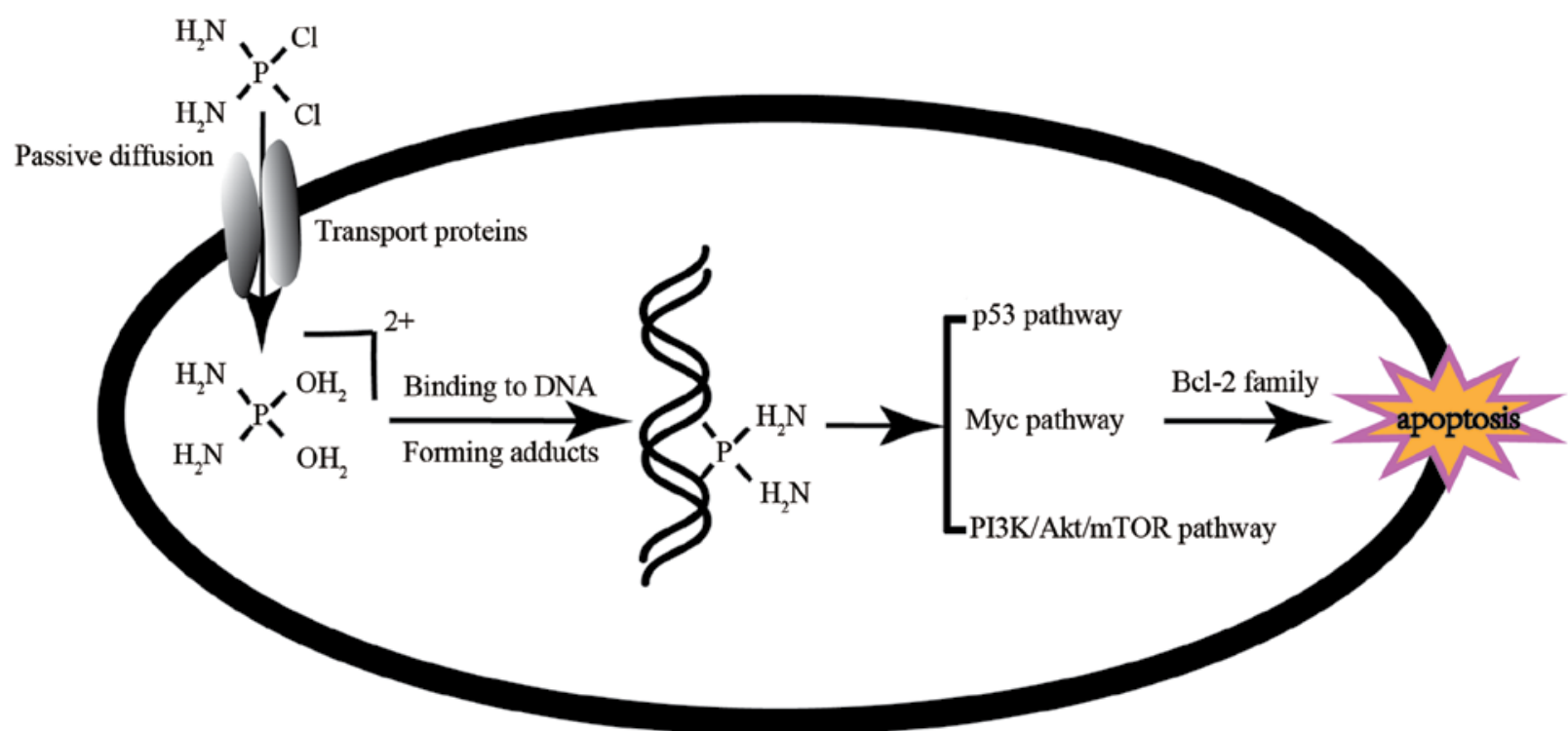

Figure 2. The main pathway of platinum functioning as an antitumor agent. Apoptosis is a terminal event in chemotherapy in ovarian cancer. Platinum enters cancer cells by passive diffusion or transport proteins and then undergoes reactions to form structures that facilitate its binding to DNA, forming DNA adducts, which activate several pathways and eventually induce mitochondrion-mediated apoptosis.

play a key role in ovarian cancer chemotherapy $(56,57)$. Bcl-2 pro-survival proteins are listed below:

i) Bcl-2 protein. $\mathrm{Bcl}-2$ was the first discovered member of the Bcl-2 family (30). The expression of Bcl-2 is intricate in ovarian cancer cells and tissues. In a previous study, by detecting Bcl-2 in a panel of 12 parent human ovarian carcinoma cell lines, the expression level of Bcl-2 was found to be negatively associated with cisplatin sensitivity. Bcl-2 protein expression was relatively low in ovarian cancer resistant cell lines (such as SKOV-3, 59M, OVCAR-3), but high in sensitive cell lines (such as $41 \mathrm{M}$ and $\mathrm{CH} 1$ ). In addition, the expression pattern of Bcl-2 also differed in various cell lines. For example, in parent cells and the cisplatin-resistant cell line, A2780, Bcl-2 was almost undetectable (52). However, another study investigating the expression of Bcl-2 protein in ovarian cancer tissues revealed a different pattern in which Bcl-2 protein expression was evidently increased in lymphatic metastasis and post-operative recurrence tissue, and was associated with tumor stage (58).
Bcl-2 knockdown by siRNA in chemoresistant multicellular spheroids of ovarian cancer has also revealed enhanced apoptosis (59). For this abnormal expression phenomenon, it is considered that apoptosis occurs due to the protein-protein interaction between pro-apoptotic factors and pro-survival factors. BAX, as a pro-apoptotic factor, can form heterodimers with pro-survival factors, such as Bcl-2 or homodimers with itself. The trend of apoptosis depends on the ratio of the two dimers (53). Therefore, the occurrence of apoptosis is not a single effect produced by one certain molecule, but a result of the participation of all molecules.

ii) $\mathrm{Mcl}-1$. Mcl-1 is, a pro-survival protein in the $\mathrm{Bcl}-2$ family. It has long been found that its upregulated expression is associated with a poor prognosis in ovarian cancer. Due to its short half-life of $\sim 30 \mathrm{~min}$ and an unstable nature, it can respond to stress conditions of cells (60). The ubiquitination of Mcl-1 is an important strategy with which to inactivate Mcl-1; thus, several ubiquitinases have been found to play a role in 
the stability of Mcl-1. In ovarian cancer cells, ubiquitinating enzymes (DUBs) can protect Mcl-1 from ubiquitination to maintain the stability of Mcl-1, At the cell and tissue level of ovarian cancer, the expression levels of Mcl-1 and DUBs have been shown to be positively associated with chemoresistance, indicating that this signal axis is attributed to chemoresistance in ovarian cancer (61). Additionally, Usp13, another ubiquitination enzyme, can also be used to stabilize Mcl-1 through ubiquitination (62). Moreover, the use of the Mcl-1 antagonist, MIM1, can effectively increase the sensitivity of ovarian cancer cells to paclitaxel (63). The increased stability of Mcl-1 seems to be the main mechanism of chemoresistance in ovarian cancer. Additionally, Mcl-1 has been found to be the direct target of some novel drugs. For example, as calcium is a universal second messenger and a significant component in ER-induced apoptosis, when inhibiting the calcium signal in ovarian cancer cells, it was found that the Mcl-1 was downregulated by calcium signal through a calmodulin-mediated pathway (64). Moreover, the overexpression of Mcl-1 was shown to reverse the apoptosis induced by ABT737, indicating a crucial hurdle of Mcl-1 in chemosensitivity (64).

iii) Bcl- $x L$. Soon after the Bcl-2 gene was cloned, Bcl-xL, with a very similar structure to Bcl-2 was identified (30). In early previous studies, Bcl-xL was found to mediate cisplatin resistance in ovarian cancer cells (65). Later studies indicated that $\mathrm{Bcl}-\mathrm{xL}$ contributes to the chemotherapeutic resistance of ovarian cancer stem cells to a greater extent than Bcl-2, as in chemotherapy-resistant ovarian cancer cells that preferentially express Bcl-xL, the $40 \%$ knockdown of $\mathrm{Bcl}-\mathrm{xL}$ expression is sufficient to fully activate caspases (49), indicating that the acquired chemoresistance of ovarian cancer is related to the abnormal increase of Bcl-xL expression.

Pro-apoptotic proteins. Pro-apoptotic proteins can be divided into 2 subgroups: The executors of apoptosis and the initiators of apoptosis, which are mainly responsible for activating the executors. The coordinated expression of both endows ovarian cancer cells with chemosensitivity. The pro-apoptotic proteins are listed below:

i) BH3-only proteins. BH3-only proteins have been assumed to be initiators of apoptosis; thus, they have to be sensitive to diverse types of cell insults. The loss of BIK deceases the antitumor effect of cisplatin by blocking the process of apoptosis in ovarian cancer (66). PUMA, another BH3-only protein, is considered to be a transcription target of p53 and an effective apoptosis-inducing factor of a number of tumor cells in recent years. It has been found that the high expression of PUMA can effectively induce the apoptosis of ovarian cancer cells by reducing the threshold set by pro-survival Bcl-xL and Mcl-1, and then increase the chemosensitivity of resistant A2780 and SKOV3 cells to cisplatin, suggesting that PUMA is an important target which may be used to overcome the chemoresistance of ovarian cancer (67). BID is considered to be the most effective initiator among BH3-only proteins. When constructed into tumor-specific oncolytic adenoviruses, the overexpression of BID exhibited great antitumor activation and enhanced the chemosensitivity to cisplatin in 9 ovarian cancer cell lines, consistent with the effect in a subcutaneous tumor xenograft model (68).

ii) $B A X, B A K$ and $B O K$. BAX, as the main effector of apoptosis, is also an important mediator of cell apoptosis.
BAK exerts similar effects on the induction of apoptosis as BAX, and BOK has been possibly less extensively studied in ovarian cancer. BAX, BAK and BOK directly integrate into the OMM using their insertion domains, affecting membrane permeability, eventually causing the downstream events of apoptosis (69). It has been demonstrated that the phosphorylation of BAX will alter its pro-apoptosis activity into pro-survival, leading to the drug resistance of ovarian cancer cells, suggesting that the change in apoptotic molecule activity is an important reason for the drug resistance of tumor cells (70). Another study found that BAX conducts the Hsp70-mediated apoptosis in ovarian cancer. Hsp70 protein can protect cells from apoptosis and decrease the activation of BAX, which can significantly attenuate the apoptosis induced by cisplatin in both resistant and sensitive cell lines. Using immunoprecipitation, it was found that Hsp70 co-immunoprecipitated with BAX in the resistant cell lines C13 and A2780cp, but not in sensitive cells (71). According to a study on ovarian cancer cells, no significant increase in the drug sensitivity of tumor cells was observed in BAX-overexpressing cells (52). However, the upregulation of BAX by BAX-expressing vectors in the ovarian cancer cell lines, SKOV3ip and DOV13, induced significant cell death (72). At the tissue level, upon analyzing the expression of BAX in 45 patients with epithelial ovarian cancer, patients with high levels of BAX in tumor tissues achieved complete sensitivity to chemotherapy, while patients with low levels of BAX did not (73). Recently, strong evidence has suggested that mitochondrial porin voltage-dependent anion channel 2 (VDAC2) is essential for BAX translocating to mitochondria-specific membranes $(74,75)$. BAK, another pro-apoptotic factor, also revealed a significant interaction with VDAC2. VDAC2 maintained BAK in an inactive conformation by interacting with its hydrophobic tail, while the absence of VDAC2 did not result in BAK activation though the threshold of apoptosis decreased (76). Some studies have demonstrated that VDAC2 plays a complex role in regulating the interaction of $\mathrm{BAX}$ and $\mathrm{BAK}$ with the mitochondria, suggesting that VDAC2 may be a promising target for regulating the activation of pro-apoptotic proteins $(77,78)$.

\section{Bcl-protein inhibitors and ovarian cancer}

The exact mechanisms of platinum therapeutic resistance remain unclear and the development of novel platinum analogues is tedious; these pose two significant issues in the clinical treatment of ovarian cancer. Platinum drugs activate a series of signaling pathways, such as p53, inducing DNA damage (79). An important target of p53 is BH3-only proteins, including Puma and Noxa (80). However, tumor cells neutralize the pro-apoptotic effects of these drugs with their high expression of Bcl-2 pro-survival proteins (81), which functions as an inhibitor of pro-apoptotic proteins. Edlich et al found that the pro-survival proteins, Bcl-xL, Bcl-2 and Mcl-1, interacted with BAX to promote BAX retrotranslocation from the mitochondria into the cytoplasm and then prevent apoptosis (82). Therefore, it is of great clinical significance to study the drugs that directly target Bcl-2 pro-survival protein. Over the past decades, the 
Table II. Promising drugs that have an impact on improving chemoresistance in ovarian cancers.

\begin{tabular}{|c|c|c|c|c|c|}
\hline Drugs & Targeting molecules & $\begin{array}{l}\text { Whether tested } \\
\text { in ovarian cancer }\end{array}$ & $\begin{array}{l}\text { Combination } \\
\text { therapy }\end{array}$ & Side-effects & (Refs.) \\
\hline ABT737 & Bcl-2, Bcl-xL, Bcl-w & Yes (in vitro and ex vivo) & Carboplatin, Cisplatin, & $\begin{array}{l}\text { Failing to be orally } \\
\text { available }\end{array}$ & $(89,91,92)$ \\
\hline ABT263 & Bcl-2, Bcl-xL, Bcl-w & Yes (in vitro and in vivo) & $\begin{array}{l}\text { Carboplatin, paclitaxel, } \\
\text { PARP inhibitor }\end{array}$ & Thrombocytopenia & $(94-96)$ \\
\hline AT101 & $\begin{array}{l}\text { Pan-proteins of } \\
\text { anti-apoptotic Bcl-2 } \\
\text { family members }\end{array}$ & Yes (in vitro) & Cisplatin & & $(100,101)$ \\
\hline ABT199 & Bcl-2 & Yes (in vitro) & Carboplatin & & $(103,104)$ \\
\hline S63845 & Mcl-1 & No & & & $(105,106)$ \\
\hline TW-37 & $\begin{array}{l}\text { Pan-proteins of } \\
\text { anti-apoptotic Bcl-2 } \\
\text { family members }\end{array}$ & Yes (in vitro) & Cisplatin & & (107) \\
\hline
\end{tabular}

identification of a variety of small molecular inhibitors of Bcl-2 pro-survival proteins has promoted their development and application in clinical practice (81). They have similar structural and functional characteristics to $\mathrm{BH} 3$ only proteins, thus, they are also termed 'BH3 mimetics' $(83,84)$ (Table II).

$A B T-737$. ABT-737 was developed through nuclear magnetic resonance (NMR)-based fragment screening and is the first classical $\mathrm{BH} 3$-mimetic that can target the 3 pro-survival proteins, Bcl-xL, Bcl-2 and Bcl-w (85). In ovarian cancer, ABT-737 induces apoptosis more potently in cisplatin-resistant ovarian cancer cells than in cisplatin-sensitive ovarian cancer cells and exerts a synergistic effect on ovarian cancer cells, indicating that ABT-737 may represent a promising therapeutic strategy in treating patients with cisplatin-resistant ovarian cancer (86). ABT-737 binds to Bcl-2 protein, resulting in the release of BAX/BAK, which can promote tumor cell apoptosis (87). The mechanisms through which ABT-737 induces apoptosis have been further studied (88). ABT-737 increases the level of DRP-1 in the mitochondria of ovarian cancer cells (88), and subsequently induces mitochondrial fission, resulting in cytochrome $c$ release, while another mechanism is that it reverses cisplatin resistance by inducing $\mathrm{Ca}^{2+}$ transfer from the ER to the mitochondrion and cytosol, resulting in the enhancement of ER- and mitochondrion-induced apoptosis (89). Furthermore, the combination therapy of cisplatin and ABT-737, can significantly increase cell death in chemo-resistant ovarian cancer cell lines (87). Most notably, previously, fresh specimens from 25 patients with advanced serous ovarian cancer (HGSOC) were exposed to ABT-737 with or without carboplatin in vitro. An antitumor effect of ABT-737 was observed, although it was not enhanced by carboplatin, indicating that the mechanism through which ABT-737 induces apoptosis in ovarian cancers in vivo is not the same as that in vitro (90) and further investigations are required.

$A B T-263$. Although ABT-737 exerts a significant promoting effect on cisplatin-induced apoptosis, it is difficult to implement in clinical applications as it is not orally available. Lately, a closely related drug, ABT-263 (Navitoclax), was identified that has been shown to enhance chemosensitivity in clinical testing $(85,91,92)$ and to reduce chemoresistance in ovarian cancer (93). In 3 HGSOC cell lines, OVCAR3, OVCAR8 and OV90, the effects of ABT-263 combined with PARP inhibitors were evaluated by detecting DNA damage accumulation, cell cycle progression, apoptosis induction and Bcl-2 family protein expression levels of tumor cells. ABT-263 alone can increase the expression of BIM, and its combination with PARP inhibitors can induce the apoptosis of tumor cells to a greater extent (94). Of note, when combined with carboplatin and paclitaxel, ABT-263 exhibited additive activity in inducing apoptosis in vitro, which provided a rationale for the treatment of ovarian cancer with ABT-263 (91). Recently, a study found that the combination of MEK inhibitor and ABT-263 significantly inhibited tumor growth in vivo and in vitro in ovarian cancer (95).

AT101. As the toxicity of Navitoclax was observed with the occurrence of dose-limiting thrombocytopenia (96) in clinical trials, there are other BH3-only mimetics, such as AT101, which is a natural product from cottonseed with a BH3-mimetic structure (97). AT101 is clinically safe and has entered phase II clinical trials. A previous study demonstrated that although AT101 can activate BAX in the induction of apoptosis, the knockdown of BAX did not completely inhibit the activation of caspase-3, while the downregulation of Smac greatly impacted the apoptosis induced by AT101, indicating that Smac release is a determinant of events in AT101-induced apoptosis, but not dependent on BAX activation (98) in ovarian cancer. In addition, in a study combining AT101 with cisplatin in the treatment of ovarian cancer cells, combination treatment evidently decreased the expression of some pro-survival proteins, such as $\mathrm{Bcl}-2$, and increased the expression of pro-apoptotic proteins, indicating great potential for overcoming chemoresistance (99).

ABT-199. The highly selective Bcl-2 inhibitor, ABT-199, which was approved by the FDA for chronic lymphocytic leukemia 
(CLL) with $17 \mathrm{p}$ deletion, has been shown to inhibit the cancer cell growth in several of cancer types (100), including ovarian cancer cells (101). Although thrombocytopenia can be avoided with the use of ABT-199, in a study characterizing the ability of ABT199, ABT-737 and other roles in ovarian cancer, ABT-737 successfully augmented carboplatin-induced tumor cell apoptosis, while ABT-199 failed to do so (102). This may be due to the phosphorylation of $\mathrm{Bcl}-2$ protein induced by chemotherapeutic drugs, which prevents the targeting of phosphorylated Bcl-2 by ABT199 and the induction of the apoptosis of the tumor cells (101). These findings remind us that efforts to explore the functional mechanisms of drugs are equally important as their discovery.

In addition, Mcl-1, functions not only as a pro-survival protein, but also as the cause of embryonic lethality in Mcl-1 knockout mice. S63845 is a specific molecular inhibitor of Mcl-1 and only affect the pro-survival function of Mcl-1 by binding to the $\mathrm{BH} 3$ domain (103). It has been reported that S63845 has a high safety dose for the treatment of cancers in mice, and is more effective in killing multiple myeloma cell lines than ABT-263 and ABT-199, while sparing the normal tissues at efficacious doses (104). TW-37 is a small-molecule inhibitor of Bcl-2 family proteins. Treatment of ovarian cancer cells with TW-37 alone or combined with cisplatin has been shown to result in the inhibition of growth and the induction ofapoptosis by downregulating the expression of Bcl-2, suggesting that TW-37 may be an efficient agent for treating ovarian cancer (105).

At present, molecular-targeted drugs that selectively target Bcl-2 family proteins have been examined in clinical trials. As early as April 2016, the selective Bcl-2 inhibitor, venetoclax (AbbVie), was approved by the FDA to be used to treat CLL due to its positive clinical results. In terms of the importance of the Bcl-2 family in chemotherapy, targeted $\mathrm{Bcl}-2$ family proteins also provide hope for the treatment of ovarian cancer (106).

\section{BH3 profiling and ovarian cancer}

Recognizing how Bcl-2 family proteins function in the apoptosis of cancer cells induced by chemotherapeutic drugs, Letai's research team (please see below) investigated an analytical technique based on Bcl-2 family proteins. Cancer cells have different expression codes among Bcl-2 family proteins. BH3-only proteins decide whether cancer cells are primed for death. In other words, BH3-only proteins can be effective predictive biomarkers, which is the rationale for $\mathrm{BH} 3$ profiling and dynamic BH3 profiling (DBP) $(107,108)$.

BH3 profiling and Bcl-2 family members. Bcl-2 family proteins are crucial regulators of apoptosis. Thus, a delicate balance between members of the Bcl-2 family determines whether the cancer cell will live or die under stress conditions (30). Pro-apoptotic proteins bind with anti-apoptotic proteins to form complexes and inhibit apoptosis. BH3-only protein is first activated as a 'guard'. Activated $\mathrm{BH} 3$-only protein can release pro-apoptotic proteins by binding with anti-apoptotic proteins, or directly activate (109), such as BAX and BAK, which aggregate together and then induce apoptosis (51). The interaction of Bcl-2 family proteins determines whether cells have the potential to go through apoptosis, indicating that the apoptotic state of cancer cells depends on not just one molecule, but the comprehensive effect of the interaction of many components. Thus, BH3 profiling technology has become an important and innovative method. Upon extracting mitochondria from cancer cells of interest and then exposing them to a $\mathrm{BH} 3$ peptide that promotes apoptosis, cytochrome $c$ release can be measured to determine MOMP. If the permeability of mitochondrial membrane in vitro increases significantly, the mitochondria are considered to be prone to apoptosis $(33,107,110)$. The term 'priming' refers to the critical condition for mitochondria to reach the apoptotic threshold. The $\mathrm{BH} 3$ profiling technique can be used to detect the threshold for apoptosis in specific cells (19). The level of the threshold determines how difficult it would be for the cells to go through apoptosis.

Performing a $\mathrm{BH} 3$ profiling assay requires the extraction of mitochondria from cells and purifying enough mitochondria requires a relatively large number of cells as experimental materials $\left(10^{7}-10^{9}\right.$ cells), which, to a certain extent, decreases the clinical operability and application. Therefore, Ryan and Letai further improved the experimental technology, based on the rationale of the $\mathrm{BH} 3$ profiling assay and proposed the dynamic BH3 profiling assay (111). The main improvement is that this method omits the procedure of extracting mitochondria, replaced by treatment with a low concentration of digoxin to not only improve the permeability of cytomembranes, but also to maintain their integrity. BH3 mimetic peptide synthesized in vitro can enter into cells and successfully interact with mitochondria. JC-1, an ideal fluorescent probe, is widely used to detect mitochondrial membrane potential (112). The degree of mitochondrial apoptosis can be evaluated by detecting the color changing of the fluorescent probe. The dynamic $\mathrm{BH} 3$ profiling assay had the same effect compared to $\mathrm{BH} 3$ profiling through comparative analysis (113). For example, Montero et al discovered that DBP successfully predicts sensitivity to chemotherapy in non-small cell lung cancer and breast cancer cell lines, and helps to select the optimal treatment strategy for patients (108).

BH3 profiling for ovarian cancer therapy. BH3 profiling and DBP assay provide novel insight into the individualized therapy for ovarian cancer. Both functional assays can be used to detect drug toxicity, predict the clinical response of ovarian cancer patients to a certain treatment, and find the best combination between drugs (108). If the response of patients to drugs can be predicted and the most optimized treatment strategy can be selected, this would greatly reduce treatment-related pain and the economic burden to patients. Fortunately, the emergence of the $\mathrm{BH} 3$ profiling assay may break this deadlock. It can be used to detect the mitochondrion priming towards antitumor drugs and predict the clinical responses to certain agents, only requiring $4 \mathrm{~h}$ from the blood draw to the profiling results (114).

It has been suggested that a number of agents may have some ability to reverse chemoresistance or induce apoptosis in ovarian cancer; however, it becomes more challenging to identify the right agents for the right patient (115). BH3 profiling and DBP assay provide practicable methods to measure how primed a cell is when treated with an antitumor agent. $\mathrm{BH} 3$ profiling was proposed to detect the proper drugs for treating patients with tumors, while DBP extends the clinical utilization of the $\mathrm{BH} 3$ profiling (115). Patients with ovarian cancer 
exhibit different responses to different drugs. DBP can also predict the chemosensitivity of ovarian cancer. For example, when using DBP to detect the priming of single-cell suspensions of samples from 16 primary ovarian adenocarcinomas and the results are consistent with the carboplatin response in ovarian cancer (116), indicating that DBP can be used in predicting the susceptibility of ovarian cancer to antitumor agents and select the most effective drugs.

Some researchers have found that the apoptotic priming of cancer cells is positively associated with the clinical response of patients to chemotherapeutic drugs. The stronger the priming, the better the prognosis. Ni et al followed 14 patients with ovarian cancer and tested their carbohydrate antigen 125 (CA125) levels to determine their response to chemotherapy and found that individuals with high apoptotic priming of mitochondria measured prior to chemotherapy had a better final response to chemotherapy (116).

$\mathrm{BH} 3$ profiling can have great application prospects for searching for novel biomarkers in future experimental research on ovarian cancer. For example, by detecting the apoptotic priming in cancer cells, the researchers found an association between the level of the OMM scaffold protein Sab in cells and the chemosensitivity of ovarian cancer cells, suggesting that Sab may be a biomarker for predicting the prognosis of ovarian cancer after chemotherapy (117). Thus, DBP or BH3 profiling can predict the chemosensitivity of patients with ovarian cancer and have great potential in medical research and individualized treatments.

\section{Conclusion}

In conclusion, the high rate of chemoresistance of ovarian cancer renders this type of cancer the most lethal among the cancers affecting women, and the dysregulation of apoptosis is one of the important mechanisms. Bcl-2 family members have a complicated interaction network and play a central role in regulating apoptosis. Recent findings revealed novel interaction relationships among Bcl-2 family members. Further research of the intricate molecular events regulating cell apoptosis would be extremely beneficial for cancer therapy, providing promising targets for overcoming the chemoresistance. Although a number of targeted drugs have entered the clinical trial stage, there are some prospective drugs that are still at the cell level, and few animal models have been established. BH3 profiling or DBP can test the the chemosensitivity of cancer cells. In the future, the combination of $\mathrm{BH} 3$ profiling or DBP and drugs targeting Bcl-2 family proteins may help to promote individualized treatments for patients with ovarian cancer.

\section{Acknowledgements}

Not applicable.

\section{Funding}

The present study was supported by the project of Hunan Provincial Natural Science Foundation of China (grant no. 2018JJ3782), the China Scholarship Fund, CSC (grant no. 201806375049), the Postgraduate Independent Exploration
And Innovation Project Of Central South University (grant nos. 2018zzts937, 2018zzts952, 2019zzts1055) and the Project of Degree and Postgraduate Education and Teaching Reform in Hunan Province (grant no. JG2018A003).

\section{Availability of data and materials}

Not applicable.

\section{Authors' contributions}

JY, HL, XJ and DZ reviewed the literature and wrote the article. SX reviewed the literature and revised the article. All authors have read and approved the final manuscript.

\section{Ethics approval and consent to participate}

Not applicable.

\section{Patient consent for publication}

Not applicable.

\section{Competing interests}

The authors declare that they have no competing interests.

\section{References}

1. Bray F, Ferlay J, Soerjomataram I, Siegel RL, Torre LA and Jemal A: Global cancer statistics 2018: GLOBOCAN estimates of incidence and mortality worldwide for 36 cancers in 185 countries. CA Cancer J Clin 68: 394-424, 2018.

2. Webb PM and Jordan SJ: Epidemiology of epithelial ovarian cancer. Best Pract Res Clin Obstet Gynaecol 41: 3-14, 2017.

3. Davis A, Tinker AV and Friedlander M: 'Platinum resistant' ovarian cancer: What is it, who to treat and how to measure benefit? Gynecol Oncol 133: 624-631, 2014.

4. International Collaborative Ovarian Neoplasm Group: Paclitaxel plus carboplatin versus standard chemotherapy with either single-agent carboplatin or cyclophosphamide, doxorubicin, and cisplatin in women with ovarian cancer: The ICON3 randomised trial. Lancet 360: 505-515, 2002.

5. Boussios S, Karihtala P, Moschetta M, Abson C, Karathanasi A, Zakynthinakis-Kyriakou N, Ryan JE, Sheriff M, Rassy E and Pavlidis N: Veliparib in ovarian cancer: A new synthetically lethal therapeutic approach. Invest New Drugs 38: 181-193, 2020.

6. Markman M: Optimizing primary chemotherapy in ovarian cancer. Hematol Oncol Clin North Am 17: 957-968, 2003.

7. Bast RC Jr, Hennessy B and Mills GB: The biology of ovarian cancer: New opportunities for translation. Nat Rev Cancer 9: 415-428, 2009.

8. Lindemann K, Gao B, Mapagu C, Fereday S, Emmanuel C, Alsop K, Traficante N, Australian Ovarian Cancer Study Group; Harnett PR, Bowtell DDL and DeFazio A: Response rates to second-line platinum-based therapy in ovarian cancer patients challenge the clinical definition of platinum resistance. Gynecol Oncol 150: 239-246, 2018.

9. Matulonis UA, Sood AK, Fallow field L, Howitt BE, Sehouli J and Karlan BY: Ovarian cancer. Nat Rev Dis Primers 2: 16061, 2016.

10. Bowtell DD, Bohm S, Ahmed AA, Aspuria PJ, Bast RC Jr, Beral V, Berek JS, Birrer MJ, Blagden S, Bookman MA, et al: Rethinking ovarian cancer II: Reducing mortality from high-grade serous ovarian cancer. Nat Rev Cancer 15: 668-679, 2015.

11. Xie H, Wang W, Xia B, Jin W and Lou G: Therapeutic applications of PARP inhibitors in ovarian cancer. Biomed Pharmacother 127: $110204,2020$. 
12. Boussios S, Karihtala P, Moschetta M, Karathanasi A, Sadauskaite A, Rassy E and Pavlidis N: Combined strategies with poly (ADP-Ribose) polymerase (PARP) inhibitors for the treatment of ovarian cancer: A literature review. Diagnostics (Basel) 9: 87, 2019.

13. Hanahan D and Weinberg RA: Hallmarks of cancer: The next generation. Cell 144: 646-674, 2011.

14. Matsuura K, Huang NJ, Cocce K, Zhang L and Kornbluth S: Downregulation of the proapoptotic protein MOAP-1 by the UBR5 ubiquitin ligase and its role in ovarian cancer resistance to cisplatin. Oncogene 36: 1698-1706, 2017.

15. Fernald $\mathrm{K}$ and Kurokawa M: Evading apoptosis in cancer. Trends Cell Biol 23: 620-633, 2013.

16. Hassan M, Watari H, AbuAlmaaty A, Ohba Y and Sakuragi N: Apoptosis and molecular targeting therapy in cancer. Biomed Res Int 2014: 150845, 2014.

17. Valentin R, Grabow S and Davids MS: The rise of apoptosis: Targeting apoptosis in hematologic malignancies. Blood 132: 1248-1264, 2018.

18. Kerr JF, Wyllie AH and Currie AR: Apoptosis: A basic biological phenomenon with wide-ranging implications in tissue kinetics. Br J Cancer 26: 239-257, 1972.

19. Chung C: Restoring the switch for cancer cell death: Targeting the apoptosis signaling pathway. Am J Health Syst Pharm 75 945-952, 2018

20. Green DR and Llambi F: Cell death signaling. Cold Spring Harb Perspect Biol 7: a006080, 2015.

21. Martinvalet D: Mitochondrial entry of cytotoxic proteases: A new insight into the granzyme B cell death pathway. Oxid Med Cell Longev 2019: 9165214, 2019.

22. Solano-Gálvez SG, Abadi-Chiriti J, Gutiérrez-Velez L, Rodríguez-Puente E, Konstat-Korzenny E, Álvarez-Hernández DA, Franyuti-Kelly G, Gutiérrez-Kobeh L and Vázquez-López R: Apoptosis: Activation and inhibition in health and disease. Med Sci (Basel) 6: 54, 2018.

23. Shamas-Din A, Kale J, Leber B and Andrews DW: Mechanisms of action of Bcl-2 family proteins. Cold Spring Harb Perspect Biol 5: a8714, 2013.

24. Caria S, Hinds MG and Kvansakul M: Structural insight into an evolutionarily ancient programmed cell death regulator- the crystal structure of marine sponge BHP2 bound to LB-Bak-2. Cell Death Dis 8: e2543, 2017.

25. Kvansakul M and Hinds MG: The Bcl-2 family: Structures, interactions and targets for drug discovery. Apoptosis 20: 136-150, 2015.

26. Banjara S, Suraweera CD, Hinds MG and Kvansakul M: The Bcl-2 family: Ancient origins, conserved structures, and divergent mechanisms. Biomolecules 10: 128, 2020.

27. Kvansakul M and Hinds MG: The structural biology of BH3-only proteins. Methods Enzymol 544: 49-74, 2014

28. Huang DC and Strasser A: BH3-Only proteins-essential initiators of apoptotic cell death. Cell 103: 839-842, 2000.

29. Elkholi R, Renault TT, Serasinghe MN and Chipuk JE: Putting the pieces together: How is the mitochondrial pathway of apoptosis regulated in cancer and chemotherapy? Cancer Metab 2: 16, 2014.

30. Adams CM, Clark-Garvey S, Porcu P and Eischen CM: Targeting the Bcl-2 family in B cell lymphoma. Front Oncol 8: 636, 2019.

31. Estaquier J, Vallette F, Vayssiere JL and Mignotte B: The mitochondrial pathways of apoptosis. Adv Exp Med Biol 942: 157-183, 2012.

32. Martinou JC and Youle RJ: Mitochondria in apoptosis: Bcl-2 family members and mitochondrial dynamics. Dev Cell 21: 92-101, 2011.

33. Letai A, Bassik MC, Walensky LD, Sorcinelli MD, Weiler S and Korsmeyer SJ: Distinct BH3 domains either sensitize or activate mitochondrial apoptosis, serving as prototype cancer therapeutics. Cancer Cell 2: 183-192, 2002.

34. Mérino D, Giam M, Hughes PD, Siggs OM, Heger K, O'Reilly LA, Adams JM, Strasser A, Lee EF, Fairlie WD and Bouillet P. The role of $\mathrm{BH} 3$-only protein bim extends beyond inhibiting Bcl-2-like prosurvival proteins. J Cell Biol 186: 355-362, 2009.

35. Sarosiek KA, Chi X, Bachman JA, Sims JJ, Montero J, Patel L, Flanagan A, Andrews DW, Sorger P and Letai A: BID preferentially activates BAK while BIM preferentially activates BAX, affecting chemotherapy response. Mol Cell 51: 751-765, 2013

36. Scorrano L, Oakes SA, Opferman JT, Cheng EH, Sorcinelli MD, Pozzan $\mathrm{T}$ and Korsmeyer SJ: BAX and BAK regulation of endoplasmic reticulum $\mathrm{Ca} 2+$ : A control point for apoptosis. Science 300: 135-139, 2003.
37. Große L, Wurm CA, Brüser C, Neumann D, Jans DC and Jakobs S: Bax assembles into large ring-like structures remodeling the mitochondrial outer membrane in apoptosis. EMBO J 35: 402-413, 2016.

38. Salvador-Gallego R, Mund M, Cosentino K, Schneider J, Unsay J, Schraermeyer U, Engelhardt J, Ries J and García-Sáez AJ: Bax assembly into rings and arcs in apoptotic mitochondria is linked to membrane pores. EMBO J 35: 389-401, 2016.

39. Smaili SS, Hsu YT, Youle RJ and Russell JT: Mitochondria in $\mathrm{Ca} 2+$ signaling and apoptosis. J Bioenerg Biomembr 32: 35-46, 2000.

40. van Zyl B, Tang D and Bowden NA: Biomarkers of platinum resistance in ovarian cancer: What can we use to improve treatment. Endocr Relat Cancer 25: R303-R318, 2018.

41. Larson CA, Blair BG, Safaei R and Howell SB: The role of the mammalian copper transporter 1 in the cellular accumulation of platinum-based drugs. Mol Pharmacol 75: 324-330, 2009.

42. Pan H, Kim E, Rankin GO, Rojanasakul Y, Tu Y and Chen YC Theaflavin-3,3'-digallate enhances the inhibitory effect of cisplatin by regulating the copper transporter 1 and glutathione in human ovarian cancer cells. Int J Mol Sci 19: 117, 2018.

43. Vousden KH and Lane DP: p53 in health and disease. Nat Rev Mol Cell Biol 8: 275-283, 2007.

44. Miyashita T and Reed JC: Tumor suppressor p53 is a direct transcriptional activator of the human bax gene. Cell 80: 293-299, 1995.

45. Conacci-Sorrell M, McFerrin L and Eisenman RN: An overview of MYC and its interactome. Cold Spring Harb Perspect Med 4: a14357, 2014.

46. Cao X, Bennett RL and May WS: c-Myc and caspase- 2 are involved in activating Bax during cytotoxic drug-induced apoptosis. J Biol Chem 283: 14490-14496, 2008

47. Hahne JC, Honig A, Meyer SR, Gambaryan S, Walter U, Wischhusen J, Häussler SF, Segerer SE, Fujita N, Dietl J and Engel JB: Downregulation of AKT reverses platinum resistance of human ovarian cancers in vitro. Oncol Rep 28: 2023-2028, 2012.

48. Cheaib B, Auguste A and Leary A: The PI3K/Akt/mTOR pathway in ovarian cancer: Therapeutic opportunities and challenges. Chin J Cancer 34: 4-16, 2015.

49. Cardenas C, Montagna MK, Pitruzzello M, Lima E, Mor G and Alvero AB: Adipocyte microenvironment promotes Bclxl expression and confers chemoresistance in ovarian cancer cells. Apoptosis 22: 558-569, 2017.

50. Adams JM and Cory S: The Bcl-2 apoptotic switch in cancer development and therapy. Oncogene 26: 1324-1337, 2007.

51. Leibowitz B and Yu J: Mitochondrial signaling in cell death via the Bcl-2 family. Cancer Biol Ther 9: 417-422, 2010.

52. Beale PJ, Rogers P, Boxall F, Sharp SY and Kelland LR: BCL-2 family protein expression and platinum drug resistance in ovarian carcinoma. Br J Cancer 82: 436-440, 2000.

53. Marx D and Meden H: Differential expression of apoptosis-associated genes Bax and $\mathrm{Bcl}-2$ in ovarian cancer. Methods Mol Med 39: 687-691, 2001

54. Fauvet R, Dufournet C, Poncelet C, Uzan C, Hugol D and Daraï E: Expression of pro-apoptotic (p53, p21, bax, bak and fas) and anti-apoptotic (Bcl-2 and $\mathrm{Bcl}-\mathrm{x})$ proteins in serous versus mucinous borderline ovarian tumours. J Surg Oncol 92: 337-343, 2005.

55. Palmer JE, Sant Cassia LJ, Irwin CJ, Morris AG and Rollason TP P53 and bcl-2 assessment in serous ovarian carcinoma. Int J Gynecol Cancer 18: 241-248, 2008.

56. Chaudhry P, Srinivasan R and Patel FD: Expression of the major fas family and Bcl-2 family of proteins in epithelial ovarian cancer (EOC) and their correlation to chemotherapeutic response and outcome. Oncol Res 18: 549-559, 2010.

57. Binju M, Amaya-Padilla MA, Wan G, Gunosewoyo H, Suryo Rahmanto Y and Yu Y: Therapeutic inducers of apoptosis in ovarian cancer. Cancers (Basel) 11: 1786, 2019.

58. Liang $M$ and Zhao J: Protein expressions of AIB1, p53 and Bcl-2 in epithelial ovarian cancer and their correlations with the clinical pathological features and prognosis. Eur Rev Med Pharmacol Sci 22: 5134-5139, 2018.

59. Yang Y, Li S, Sun Y, Zhang D, Zhao Z and Liu L: Reversing platinum resistance in ovarian cancer multicellular spheroids by targeting Bcl-2. Onco Targets Ther 12: 897-906, 2019.

60. Inuzuka H, Shaik S, Onoyama I, Gao D, Tseng A, Maser RS, Zhai B, Wan L, Gutierrez A, Lau AW, et al: SCF(FBW7) regulates cellular apoptosis by targeting MCL1 for ubiquitylation and destruction. Nature 471: 104-109, 2011. 
61. Wu X, Luo Q, Zhao P, Chang W, Wang Y, Shu T, Ding F, Li B and Liu Z: MGMT-activated DUB3 stabilizes MCL1 and drives chemoresistance in ovarian cancer. Proc Natl Acad Sci USA 116: 2961-2966, 2019

62. Zhang S, Zhang M, Jing Y, Yin X, Ma P, Zhang Z, Wang X, Di W and Zhuang G: Deubiquitinase USP13 dictates MCL1 stability and sensitivity to BH3 mimetic inhibitors. Nat Commun 9: 215, 2018.

63. Habata S, Iwasaki M, Sugio A, Suzuki M, Tamate M, Satohisa S, Tanaka R and Saito T: BAG3-mediated Mcl-1 stabilization contributes to drug resistance via interaction with USP9X in ovarian cancer. Int J Oncol 49: 402-410, 2016.

64. Bonnefond ML, Lambert B, Giffard F, Abeilard E, Brotin E, Louis MH, Gueye MS, Gauduchon P, Poulain L and N'Diaye M: Calcium signals inhibition sensitizes ovarian carcinoma cells to anti-Bcl-xL strategies through Mcl-1 down-regulation. Apoptosis 20: 535-550, 2015.

65. Liu JR, Fletcher B, Page C, Hu C, Nunez G and Baker V: Bcl-xL is expressed in ovarian carcinoma and modulates chemotherapy-induced apoptosis. Gynecol Oncol 70: 398-403, 1998.

66. Nawrocki ST, Kelly KR, Smith PG, Espitia CM, Possemato A, Beausoleil SA, Milhollen M, Blakemore S, Thomas M, Berger A and Carew JS: Disrupting protein NEDDylation with MLN4924 is a novel strategy to target cisplatin resistance in ovarian cancer. Clin Cancer Res 19: 3577-3590, 2013.

67. Yuan Z, Cao K, Lin C, Li L, Liu HY, Zhao XY, Liu L, Deng HX, Li J, Nie CL and Wei YQ: The p53 upregulated modulator of apoptosis (PUMA) chemosensitizes intrinsically resistant ovarian cancer cells to cisplatin by lowering the threshold set by Bcl-x(L) and Mcl-1. Mol Med 17: 1262-1274, 2011.

68. Dai Y, Zhao XJ, Li F, Yuan Y, Yan DM, Cao H, Huang XY, Hu Z, Ma D and Gao QL: Truncated Bid regulates cisplatin response via activation of mitochondrial apoptosis pathway in ovarian cancer. Hum Gene Ther 31: 325-338, 2020

69. Yamaguchi H, Bhalla K and Wang HG: Bax plays a pivotal role in thapsigargin-induced apoptosis of human colon cancer HCT116 cells by controlling Smac/Diablo and Omi/HtrA2 release from mitochondria. Cancer Res 63: 1483-1489, 2003.

70. Kale J, Kutuk O, Brito GC, Andrews TS, Leber B, Letai A and Andrews DW: Phosphorylation switches Bax from promoting to inhibiting apoptosis thereby increasing drug resistance. EMBO Rep 19: e45235, 2018

71. Yang X, Wang J, Zhou Y, Wang Y, Wang S and Zhang W: Hsp70 promotes chemoresistance by blocking Bax mitochondrial translocation in ovarian cancer cells. Cancer Lett 321: 137-143, 2012.

72. Huang X, Lin T, Gu J, Zhang L, Roth JA, Stephens LC, Yu Y, Liu J and Fang B: Combined TRAIL and Bax gene therapy prolonged survival in mice with ovarian cancer xenograft. Gene Ther 9: 1379-1386, 2002.

73. Tai YT, Lee S, Niloff E, Weisman C, Strobel T and Cannistra SA: BAX protein expression and clinical outcome in epithelial ovarian cancer. J Clin Oncol 16: 2583-2590, 1998.

74. Lauterwasser J, Todt F, Zerbes RM, Nguyen TN, Craigen W, Lazarou M, van der Laan M and Edlich F: The porin VDAC2 is the mitochondrial platform for Bax retrotranslocation. Sci Rep 6: 32994, 2016

75. Edlich F: BCL-2 proteins and apoptosis: Recent insights and unknowns. Biochem Biophys Res Commun 500: 26-34, 2018.

76. Lazarou M, Stojanovski D, Frazier AE, Kotevski A, Dewson G, Craigen WJ, Kluck RM, Vaux DL and Ryan MT: Inhibition of Bak activation by VDAC2 is dependent on the Bak transmembrane anchor. J Biol Chem 285: 36876-36883, 2010.

77. Shoshan-Barmatz V, Keinan $\mathrm{N}$ and Zaid $\mathrm{H}$ : Uncovering the role of VDAC in the regulation of cell life and death. J Bioenerg Biomembr 40: 183-191, 2008.

78. Shimizu S, Narita M and Tsujimoto Y: Bcl-2 family proteins regulate the release of apoptogenic cytochrome $c$ by the mitochondrial channel VDAC. Nature 399: 483-487, 1999.

79. Boulikas T and Vougiouka M: Cisplatin and platinum drugs at the molecular level (Review). Oncol Rep 10: 1663-1682, 2003.

80. Li J, Lee B and Lee AS: Endoplasmic reticulum stress-induced apoptosis: Multiple pathways and activation of p53-up-regulated modulator of apoptosis (PUMA) and NOXA by p53. J Biol Chem 281: 7260-7270, 2006.

81. Campbell KJ and Tait S: Targeting BCL-2 regulated apoptosis in cancer. Open Biol 8: 180002, 2018.

82. Edlich F, Banerjee S, Suzuki M, Cleland MM, Arnoult D, Wang C, Neutzner A, Tjandra $\mathrm{N}$ and Youle RJ: Bcl-x(L) retrotranslocates Bax from the mitochondria into the cytosol. Cell 145: 104-116, 2011.
83. Delbridge AR and Strasser A: The BCL-2 protein family, BH3-mimetics and cancer therapy. Cell Death Differ 22: 1071-1080, 2015.

84. Billard C: $\mathrm{BH} 3$ mimetics: Status of the field and new developments. Mol Cancer Ther 12: 1691-1700, 2013.

85. Oltersdorf T, Elmore SW, Shoemaker AR, Armstrong RC, Augeri DJ, Belli BA, Bruncko M, Deckwerth TL, Dinges J, Hajduk PJ, et al: An inhibitor of Bcl-2 family proteins induces regression of solid tumours. Nature 435: 677-681, 2005.

86. Xu Y, Gao W, Zhang Y, Wu S, Liu Y, Deng X, Xie L, Yang J, $\mathrm{Yu} \mathrm{H}, \mathrm{Su} \mathrm{J}$ and Sun L: ABT737 reverses cisplatin resistance by targeting glucose metabolism of human ovarian cancer cells. Int J Oncol 53: 1055-1068, 2018

87. Dai Y, Jin S, Li X and Wang D: The involvement of Bcl-2 family proteins in AKT-regulated cell survival in cisplatin resistant epithelial ovarian cancer. Oncotarget 8: 1354-1368, 2017.

88. Yu Y, Xu L, Qi L, Wang C, Xu N, Liu S, Li S, Tian H, Liu W, Xu Y and Li Z: ABT737 induces mitochondrial pathway apoptosis and mitophagy by regulating DRP1-dependent mitochondrial fission in human ovarian cancer cells. Biomed Pharmacother 96: 22-29, 2017.

89. Xie Q, Su J, Jiao B, Shen L, Ma L, Qu X, Yu C, Jiang X, Xu Y and Sun L: ABT737 reverses cisplatin resistance by regulating ER-mitochondria $\mathrm{Ca} 2+$ signal transduction in human ovarian cancer cells. Int J Oncol 49: 2507-2519, 2016.

90. Lheureux S, N'Diaye M, Blanc-Fournier C, Dugué AE, Clarisse B, Dutoit S, Giffard F, Abeilard E, Briand M, Labiche A, et al: Identification of predictive factors of response to the BH3-mimetic molecule ABT-737: An ex vivo experiment in human serous ovarian carcinoma. Int J Cancer 136: E340-E350, 2015.

91. Stamelos VA, Robinson E, Redman CW and Richardson A Navitoclax augments the activity of carboplatin and paclitaxel combinations in ovarian cancer cells. Gynecol Oncol 128: 377-382, 2013

92. Tse C, Shoemaker AR, Adickes J, Anderson MG, Chen J, Jin S, Johnson EF, Marsh KC, Mitten MJ, Nimmer P, et al: ABT-263: A potent and orally bioavailable Bcl-2 family inhibitor. Cancer Res 68: 3421-3428, 2008

93. Wong M, Tan N, Zha J, Peale FV, Yue P, Fairbrother WJ and Belmont LD: Navitoclax (ABT-263) reduces Bcl-x(L)-mediated chemoresistance in ovarian cancer models. Mol Cancer Ther 11: 1026-1035, 2012.

94. Yokoyama T, Kohn EC, Brill E and Lee JM: Apoptosis is augmented in high-grade serous ovarian cancer by the combined inhibition of Bcl-2/Bcl-xL and PARP. Int J Oncol 50: 1064-1074, 2017.

95. Iavarone C, Zervantonakis IK, Selfors LM, Palakurthi S, Liu JF, Drapkin R, Matulonis UA, Hallberg D, Velculescu VE, Leverson JD, et al: Combined MEK and BCL-2/XL inhibition is effective in high-grade serous ovarian cancer patient-derived xenograft models and bim levels are predictive of responsiveness. Mol Cancer Ther 18: 642-655, 2019.

96. Wilson WH, O'Connor OA, Czuczman MS, LaCasce AS, Gerecitano JF, Leonard JP, Tulpule A, Dunleavy K, Xiong H, Chiu YL, et al: Navitoclax, a targeted high-affinity inhibitor of BCL-2, in lymphoid malignancies: A phase 1 dose-escalation study of safety, pharmacokinetics, pharmacodynamics, and antitumour activity. Lancet Oncol 11: 1149-1159, 2010.

97. Meng Y, Tang W, Dai Y, Wu X, Liu M, Ji Q, Ji M, Pienta K, Lawrence $\mathrm{T}$ and $\mathrm{Xu} \mathrm{L}$ : Natural $\mathrm{BH} 3$ mimetic (-)-gossypol chemosensitizes human prostate cancer via Bcl-xL inhibition accompanied by increase of Puma and Noxa. Mol Cancer Ther 7: 2192-2202, 2008

98. Hu W, Wang F, Tang J, Liu X, Yuan Z, Nie C and Wei Y: Proapoptotic protein Smac mediates apoptosis in cisplatin-resistant ovarian cancer cells when treated with the anti-tumor agent AT101. J Biol Chem 287: 68-80, 2012.

99. Karaca B, Atmaca H, Bozkurt E, Kisim A, Uzunoglu S, Karabulut B, Sezgin C, Sanli UA and Uslu R: Combination of AT-101/cisplatin overcomes chemoresistance by inducing apoptosis and modulating epigenetics in human ovarian cancer cells. Mol Biol Rep 40: 3925-3933, 2013.

100. Touzeau C, Dousset C, Le Gouill S, Sampath D, Leverson JD Souers AJ, Maïga S, Béné MC, Moreau P, Pellat-Deceunynck C andAmiot M: The Bcl-2 specific BH3 mimetic ABT-199: A promising targeted therapy for $\mathrm{t}(11 ; 14)$ multiple myeloma. Leukemia 28: 210-212, 2014.

101. Song T,Zhang M,Liu P, Xue Z, Fan Y and Zhang Z: Identification of JNK1 as a predicting biomarker for ABT-199 and paclitaxel combination treatment. Biochem Pharmacol 155: 102-109, 2018 
102. Abed MN, Abdullah MI and Richardson A: Antagonism of $\mathrm{Bcl}-\mathrm{XL}$ is necessary for synergy between carboplatin and BH3 mimetics in ovarian cancer cells. J Ovarian Res 9: 25, 2016.

103. Yamaguchi R, Lartigue L and Perkins G: Targeting Mcl-1 and other Bcl-2 family member proteins in cancer therapy. Pharmacol Ther 195: 13-20, 2019.

104. Kotschy A, Szlavik Z, Murray J, Davidson J, Maragno AL, Toumelin-Braizat GL, Chanrion M, Kelly GL, Gong JN, Moujalled DM, et al: The MCL1 inhibitor S63845 is tolerable and effective in diverse cancer models. Nature 538: 477-482, 2016.

105. Wang H, Zhang Z, Wei $X$ and Dai R: Small-molecule inhibitor of Bcl-2 (TW-37) suppresses growth and enhances cisplatin-induced apoptosis in ovarian cancer cells. J Ovarian Res 8: 3, 2015.

106. Deng J: How to unleash mitochondrial apoptotic blockades to kill cancers? Acta Pharm Sin B 7: 18-26, 2017.

107. Certo M, Del Gaizo Moore V, Nishino M, Wei G, Korsmeyer S, Armstrong SA and Letai A: Mitochondria primed by death signals determine cellular addiction to antiapoptotic BCL-2 family members. Cancer Cell 9: 351-365, 2006.

108. Montero J, Sarosiek KA, DeAngelo JD, Maertens O, Ryan J, Ercan D, Piao H, Horowitz NS, Berkowitz RS, Matulonis U, et al: Drug-induced death signaling strategy rapidly predicts cancer response to chemotherapy. Cell 160: 977-989, 2015.

109. Kim H, Tu HC, Ren D, Takeuchi O, Jeffers JR, Zambetti GP, Hsieh JJD and Cheng EHY: Stepwise activation of BAX and BAK by tBID, BIM, and PUMA initiates mitochondrial apoptosis. Mol Cell 36: 487-499, 2009.

110. Deng J, Carlson N, Takeyama K, Dal Cin P, Shipp M and Letai A: BH3 profiling identifies three distinct classes of apoptotic blocks to predict response to ABT-737 and conventional chemotherapeutic agents. Cancer Cell 12: 171-185, 2007.
111. Ryan J and Letai A: BH3 profiling in whole cells by fluorimeter or FACS. Methods 61: 156-164, 2013.

112. Elefantova K, Lakatos B, Kubickova J, Sulova Z and Breier A Detection of the mitochondrial membrane potential by the cationic dye JC-1 in L1210 cells with massive overexpression of the plasma membrane ABCB1 drug transporter. Int J Mol Sci 19: 1985, 2018.

113. Ryan J, Montero J, Rocco J and Letai A: iBH3: Simple, fixable $\mathrm{BH} 3$ profiling to determine apoptotic priming in primary tissue by flow cytometry. Biol Chem 397: 671-678, 2016.

114. Del Gaizo Moore V and Letai A: BH3 profiling-measuring integrated function of the mitochondrial apoptotic pathway to predict cell fate decisions. Cancer Lett 332: 202-205, 2013.

115. Montero J and Letai A: Dynamic BH3 profiling-poking cancer cells with a stick. Mol Cell Oncol 3: e1040144, 2016.

116. Ni CT, Sarosiek KA, Vo TT, Ryan JA, Tammareddi A, Del Gaizo Moore V, Deng J, Anderson KC, Richardson P, Tai YT, et al: Pretreatment mitochondrial priming correlates with clinical response to cytotoxic chemotherapy. Science 334: 1129-1133, 2011.

117. Paudel I, Hernandez SM, Portalatin GM, Chambers TP and Chambers JW: Sab concentrations indicate chemotherapeutic susceptibility in ovarian cancer cell lines. Biochem J 475: 3471-3492, 2018.

This work is licensed under a Creative Commons Attribution-NonCommercial-NoDerivatives 4.0 International (CC BY-NC-ND 4.0) License. 